\title{
Catalytic/Bio-catalytic and Novel Processes for Sustainable Development
}

\author{
Said SHE Elnashaie* \\ Department of Chemical and Biological Engineering, University of British Columbia, Vancouver, Canada
}

*Corresponding author: Said SHE Elnashaie, Department of Chemical and Biological Engineering, University of British Columbia, Vancouver, Canada.
Received Date: August 07, 2019

Published Date: August 30, 2019

\section{Summary}

Coupling experimental techniques to mathematical modeling, computer simulation and optimization using verified reliable models has opened the door for excellent improvements in classical processes and introducing novel processes to achieve not only clean environment but also Sustainable Development (SD) where Environmental Engineering (EE) is a subsystem of SD. The Integrated System Approach (ISA) based on System Theory (ST) is the best technique to approach this complicated system and achieve their aims. The best approach is through integration of biochemical and thermo-catalytic processes to produce both Bio-Fuels (BFs) and Bio-Products (BPs) using Renewable Raw Materials (RRMs). This is a challenge to design and optimize Integrated Bio-Refineries (IBRs) using RRMs to replace classical processes using non-RRMs. Many of these processes show bifurcation/instability phenomena and these phenomena will not be addressed in this paper. The paper will address the above challenges and related ones, starting with general simplified description of SD Engineering (SDE) followed by Chemical Reaction Engineering (CRE) then Biochemical Reaction Engineering Processes (BREPs) and closing up with Thermo-Catalytic Reaction Processes (TCRPs) to complete the integrated network at the present state of knowledge..

Abbreviation: SD: Sustainable Development; EE: Environmental Engineering; ISA: Integrated System Approach; ST: System Theory; BFs: BioFuels; BPs: Bio-Products; RRMs: Renewable Raw Materials; IBRs: Integrated Bio-Refineries; SDE: Sustainable Development Engineering; CRE: Chemical Reaction Engineering; BREPs: Biochemical Reaction Engineering Processes; TCRPs: Thermo-Catalytic Reaction Processes

\section{Introduction}

In the last 2-3 decades the world has been witnessing an extensive wave of Multi-Disciplinary (MD) [or Cross-Disciplinary (CD)] research to advance in an extensive way all kinds of technologies. The main challenge is to introduce different kinds of techniques to radically improve the efficiency of different processes and introduce new processes relying on novel RRMs in order to achieve SD. This radical change from exhaustible non-RRMs to nonexhaustible RRMs requires new novel technologies to handle these new and novel RRMs. Not only that but the researches in these directions opened the doors wide open for challenging some of the earlier processes ceilings such as the thermo-dynamic equilibrium and product ceilings using membrane reactors and bio-reactors as well as other techniques.

The integration of extensive use of experimental techniques coupled to mathematical modeling, computer simulation and optimization using verified reliable models has opened the door wide open for an excellent improvement in classical processes as well as novel processes in order to achieve not only SD but also clean environment; where EE is a subsystem of SD. The ISA based on modern ST is the best technique to approach these complicated systems and achieve their aims. SD can be considered formed of a technological subsystem that we call SDE and other subsystems such as: politics, economics, socioeconomics, habits, consumers taste, etc. While SDE can be considered a system formed of EE and RRMs. Catalytic and Bio-catalytic processes are at the heart of these challenges and their best integration is through combining biochemical and thermo-catalytic processes to produce both BFs and BPs using RRMs to achieve SD. This is a challenge to design and optimize IBRs using RRMs to replace Petroleum Refineries (PRs), Petro-Chemical Complexes (PCCs) and other Chemical Industries (CIs) using non-RRMs and therefore not achieving SD.

IBRs are formed each of at least two platforms, one biochemical based on sugar(s) which are obtained from RRMs such 
as lignocellulose, which goes through a number of steps such as lignocellulose fractionation to remove the lignin from the cellulose and hemicellulose; then both are hydrolyzed to obtain the sugars which are then fermented to produce a wide range of BFs and BPs. The other thermo-catalytic platform is based on syngas $\left(\mathrm{H}_{2}\right.$, $\mathrm{CO}$ and $\mathrm{CO}_{2}$ ) usually obtained from gasification of RRMs such as lignocellulose. $\mathrm{CO}_{2}$ is removed from the syngas and the $\mathrm{H}_{2}$ and $\mathrm{CO}$ mixture is mostly used in the production of a wide range BFs and BPs using the very promising and commercially successful process since the Second World War, the catalytic Fischer Tropsch (FT) processes. Many of the above technologies and also many important industrial catalytic processes such as Fluid Catalytic Cracking (FCC) for the production of high-octane number gasoline and the UNIPOL process for the production of polyethylene from ethylene in the gas phase under low pressure and similar processes show bifurcation and instability phenomena.

If the Bio-Refinery (BR) is formed of one platform then it is not an IBR but rather an Elementary Bio-Refinery (EBR). In both cases the BR can be producing direct energy without considering this a platform. The IBRs with at least two platforms are not the end of the story for it is possible to have IBRs with three platforms where the third platform is an algae platform; where microalgae is growing in a photo-bioreactor and the produced algae gives about $50 \%$ of its weight as biodiesel and the remaining is lignocellulose that can be used in the sugar platform and/or the thermo-catalytic platform. Such interaction between the different platforms of IBRs is one of their most important and promising characteristics.

The challenges addressed in this paper do not include only novel technologies for RRMs but also clean efficient technologies for both RRMs and non-RRMs. This will not include only numerical/ experimental investigations for well-established configurations but also exploration of conceptual optimization including novel configurations/modes of operation for catalytic and bio-catalytic reactors. This includes moving from fixed bed configuration for catalytic reactors where the effectiveness factor $(\eta)$ in a fixed bed is very small (e.g.: for fixed bed catalytic steam reformer, $\eta=10-2$ $10-3$ ) to a fluidized bed where $\eta=1.0$, thus increasing the rate of reaction tremendously but does not improve the thermodynamic equilibrium ceiling. Other techniques are used to shift the thermodynamic ceiling in what we call sequential de-bottle-necking. It is the use of hydrogen selective membranes that is the best way to shift thermodynamic equilibrium ceiling. Other challenges include membrane reactors with dehydrogenation in one side of the membrane and a hydrogenation on the other side, which does not only achieve shifting the thermodynamic equilibrium ceiling; but also, through the choice of a suitable membrane, achieves overall auto-thermicity of the reactor because dehydrogenation reactions are always endothermic and hydrogenation reactions are always exothermic. One example is catalytic dehydrogenation of Ethyl benzene to Styrene in one side and Hydrogenation of Nitrobenzene to Aniline in the other side of the membrane.

These different kinds of challenges concerning reactor design does not decrease the importance of the catalysis side, development of new catalysts with higher activity, better selectivity to desired products, higher stability and resistance to catalyst deactivation. In addition to the effect of the type of reactor on the required characteristics of the catalyst (e.g.: catalyst resistance to attrition when used in fluidized bed), also material science is important on other sides such as hydrogen selective membranes and other design aspects. Since new work, with new RRMs and clean technology requires a strong integration of experimental and mathematical work, it is a must for both catalytic and bio-catalytic reactors to have reliable kinetic models to use in the mathematical modeling and simulation of the results of experimental reactors, verify the models and use the verified reliable models for design and optimization with minimum trial and error, not only numerical optimization but also conceptual optimization regarding novel configurations and modes of operation.

The bio-catalytic reactors present similar challenges but in a different way. They can be divided into enzyme bio - reactors where the enzyme is the bio - catalyst that catalyzes the bio-reaction(s) but does not grow with the reaction(s). The other types are bioreactors where the biocatalyst is a micro-organism that does not only catalyze the reaction(s) but also grew during the progress of the bio-reaction(s). Both types of bio - catalysts can be free in solution or immobilized. The immobilization makes the catalyst more stable inside the bio - reactor and allows the use of high flow rates without washing the bio - catalyst out of the reactors. Some bio - catalysts have monotonic kinetics where the rate of reaction increases with the increase of substrate concentration till it reaches a maximum saturation value and does not further increase or decrease. Other bio-catalysts can also have non-monotonic kinetics where the rate of reaction increases with the increase of substrate concentration till it reaches a maximum value at an optimum substrate concentration and then further increase of the substrate concentration causes the rate of bio-reaction to decrease till it reaches a lowest value asymptotically. This non-monotonic kinetics can case bifurcation behavior that affects not only the design but also the stability and the control of the bio - reactor. The rate of reaction may also be inhibited by the product (e.g.: bio-ethanol production by fermentation, the rate of fermentation reaches almost zero when the product bioethanol reaches about 10\%). This product inhibition can be conquered through continuous removal of the inhibiting product (e.g.: per-vaporation membranes for continuous removal of bio - ethanol produced by fermentation of sugars). Non-monotonic kinetics and product inhibition are also possible for catalytic reactions.

\section{Sustainable Development (SD)}

The best approach to handle SD, which is one step ahead of EE is the ISA based on ST. This is due to the fact that this relatively more modern approach manages to organize SD, SDE, EE and RRMs in the correct structure and relate them correctly to each other. ISA is very useful in both research and education especially in the emerging extremely important MD education, training and research fields. ISA and MD research are essential for innovation in all scientific and engineering fields with SD added to the optimization objectives. 
Terminology used will be mainly that based on nonlinear dynamics and classification is that based on the general thermodynamics' basis. In addition to a clear distinction between the system, its environment and the boundaries between them. When the system is formed of a number of interacting subsystems then the interaction between the subsystems and the +/- synergy of these interactions are essential to recognize the properties and characteristics of the system which is not simply the addition of the properties and characteristics of these sub-systems.

\section{System theory (ST) and integrated system approach (ISA)}

The main theme of this part of the paper is to stress the importance of the ST/ISA when dealing with issues of SD, SDE, EE, BFs, BPs and IBRs. ST is a basic tool for dealing with these critical issues. It should be used in engineering education and research. Definitions of some important terms are essential.

a. What is a system?

System originates from the word systema in Greek. It means an assemblage of objects related to each other by some interaction or interdependence. A more pragmatic description regarding systems includes the following:

- It is a complex composed of parts (elements or subsystems).

- $\quad$ Systems, subsystems and element are relative and depend upon the degree of analysis.

- The subsystem/elements of the system can be parts of the system or of the processes or both, i.e. parts of the industrial FCC Units .They are formed of: a reactor and a regenerator combined to form the unit each of the two parts has number of processes taking place within its boundaries.

- The properties of the system are non-linear interaction of the properties of its sub-systems.

b. The State of the system (SS) and state variables (SVs)

- SVs define the SS and are chosen according to the nature of the system.

c. Input variables (input parameters)

They are external to the system and not SVs; they can be inputs or parameters for rates of processes. For example, the feed temperature and composition of the feed stream to a distillation tower or a chemical reactor or the feed temperature to a heat exchanger are input variables (parameters); also, the rate constant of a reaction is a parameter, etc.

\section{d. Design variables (design parameters)}

They are associated with the design of the system and are usually fixed. Examples are the diameter and height of a Continuous Stirred Tank Reactor (CSTR) or of a tubular reactor, etc.

e. Boundaries of system
They distinguish the system from its environment. The relation between the system and its environment leads to one of the most important classifications of systems:

- Isolated systems: Do not exchange matter nor energy with the environment. They tend to the state of thermodynamic equilibrium. An example is a batch adiabatic reactor.

- Closed systems: Do not exchange matter with the environment but do exchange energy. It tends to thermodynamic equilibrium. Batch non-adiabatic reactor is an example.

- Open systems: Exchange matter and energy with the environment. Do not tend to thermodynamic equilibrium but to steady state or what should better be called a "stationary non-equilibrium state", characterized by minimum entropy generation, called steady state in chemical engineering. A CSTR is an example.

Therefore, the term steady state commonly used in chemical/ biological engineering and other disciplines is not accurate. A more meaningful rigorous term should be "stationary non-equilibrium state". This steady state of lumped systems is a point in a space having the same dimensions as the problem (number of components +temperature + pressure, etc.); whereas for distributed systems it is a profile in the space coordinate(s) as additional dimension(s). Unsteady state of an open system starts at an initial condition and tends with time towards a steady state when the system is stable (a point for lumped system and profile for distributed systems). And reach a certain attractor (periodic or chaotic) when the system is unstable.

\section{ISA}

Limited success has been achieved in SDE and of course in SD and related sub-systems. However, the potential to achieve great successes is quite large. This requires the use of an ISA based on ST to tackle complexity/ bio-complexity and related challenges and couple that to MD. On the technical front a number of advances; experimentally, theoretically, modeling and numerically are already improving the usefulness of models coupled to laboratory and pilot plant experiments. To maximize the usefulness of this MD approach rigorous methodologies must be used for dealing with missing and uncertain information; improved methods for interpretation of multivariate data sets and for multi-objective decision-making involving trade-offs among conflicting goals and novel modeling methods as alternatives to traditional mathematical models. More generally, there is a great need for operational definitions and metrics for sustainability and resilience in economic, ecological, and societal systems. SD in a changing global environment requires resilience at many levels, including human communities and economic enterprises. In the face of ever-increasing global complexity and volatility, it is essential to move beyond a simplistic steady state model of sustainability but also develop dynamic/ adaptive policies and strategies that enable societal and industrial institutions to cope with unexpected challenges, balancing needs to achieve an efficient SD. 


\section{Basic principles of SD, SDE and BFs}

Simple analysis in the introduction and other parts of this short paper highlight the following basic principles:

1. SD is a system formed of technological and nontechnological subsystems as shown in Figure 1 and detailed in this part of the paper, with special emphasis on the technological subsystem which is SDE with the other subsystems in the background.

2. As illustrated in Figure 2, SDE is a subsystem of the technological subsystem of SD.

3. SD can also be divided into:

a. SD with respect to production, which is the main emphasis of this paper

b. SD with respect to consumption, which is only in the background of this paper

Sustainable Production and Consumption (SP \& C) emerged as a key issue on the SD agenda at the United Nations Conference on Environment and Development (UNCED) in Rio de Janeiro on 1992. The agenda called on governments, businesses, and others to implement measures to promote efficiencies in production and encourage sustainable patterns of consumption. It went on to say that the developed countries should take the lead in introducing those measures. An international agenda had been introduced by the UN Commission on Sustainable Development (UNCSD), in cooperation with national governments; the Organization for Economic Cooperation and Development (OECD) and others responded to this mandate with an international work program and recommendations for action. Proposed action items included the following:

- $\quad$ Pricing reforms to internalize environmental costs and remove subsidies that generate unsustainable consumption; however, this may have negative social impacts on poor classes in many countries

- $\quad$ Green public procurement policies a. UNCSD requested businesses to do the following:

1. Make environmental criteria an integral part of their purchasing policies

2. Introduce more efficient processes to produce environmentally friendly products using clean RRMs

3. Have durable products with long life-spans

4. Improve the after sale and recycle characteristics of products and promote sustainable consumption through advertising, marketing, and product information

- Efficient engineering is a subsystem of EE, which is a subsystem of SDE, thus it is necessary but not sufficient for EE, which itself is necessary but not sufficient for SDE aiming at sustainability.

- $\quad$ Metrics are necessary for measuring sustainability; they can be divided into three groups: environmental indicators, economic indicators, and social indicators. This emphasizes the fact that EE is necessary but not sufficient for sustainability. However, the three categories do not show explicitly the importance of RRMs for sustainability, shown very simply and briefly above.

- $\quad$ RRMs represent a crucial component of SDE and, thus, SD. Green technology based on non-RRMs may be satisfactory from an EE point of view but is not sustainable.

- $\quad$ BFs that are Renewable Clean Fuels (RCF) represent an important subsystem of Renewable Clean Energy (RCE), which is itself an important subsystem of SDE and, thus, SD. Under the Energy Policy Act of 2005, the Environmental Protection Agency (EPA) in the USA is responsible for promulgating regulations to ensure that gasoline sold in the USA contains a minimum volume of RCFs. A national renewable fuel program (also known as the renewable fuel standard program, or RFS program) will increase the volume of RCFs required to be blended into gasoline, starting with 4.0 billion gallons in calendar year 2006 and nearly doubled to 7.5 billion gallons in 2012. The RFS program was developed in collaboration with refineries, RCFs producers, and many other stakeholders (U.S.EPA, n.d.; see also http://www.wbfevent.com/).

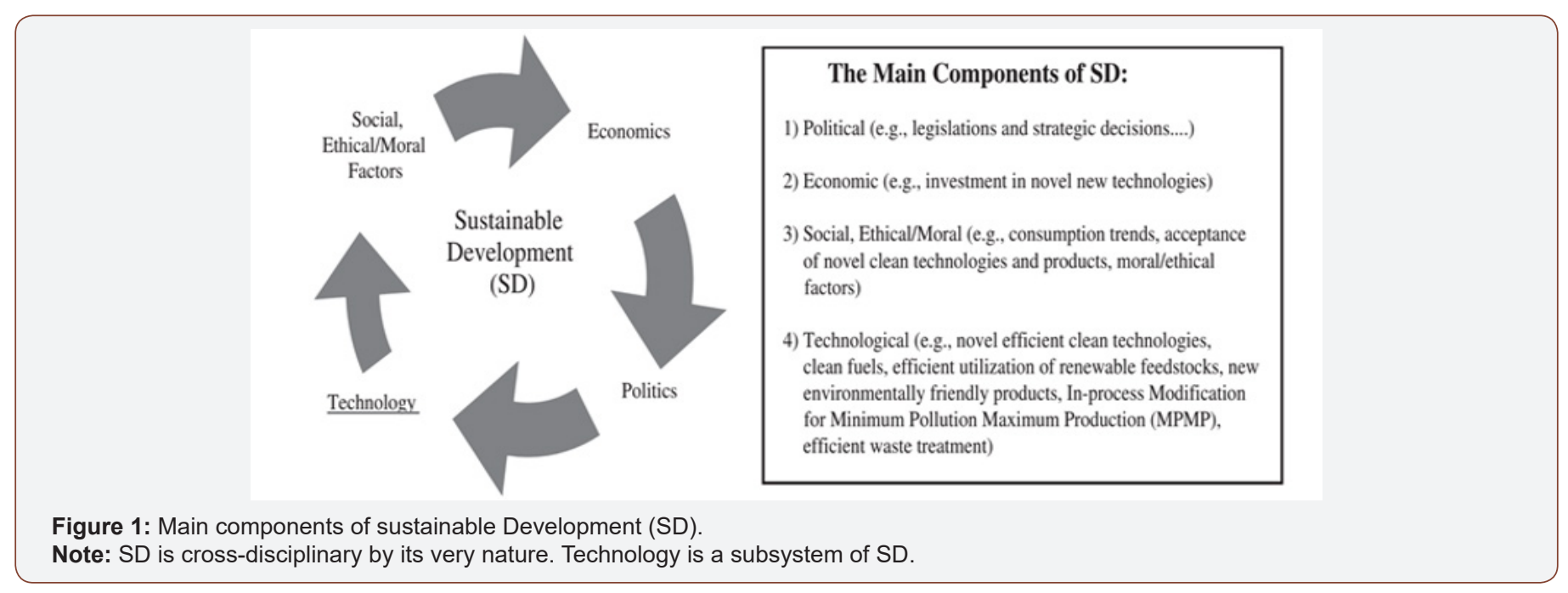


- BFs represent important subsystem of RCFs, which is the main emphasis of this part of the paper with other subsystems in the background. There is a large number of BFs; each can be produced through different routes. Figure 3 shows some of these routes for bio-diesel and bio-hydrogen, while Figure 4 shows routes to bio-ethanol. All will have their positions in the clean fuels matrix of the future.Figure 3 shows two types of biodiesels, the strategic Fischer Tropsch (FT) bio-diesel and the non-strategic bio-diesel from trans-esterification of vegetable oils. A third type, which may prove to be more strategic than both, is the algae biodiesel (Figure 1-4).

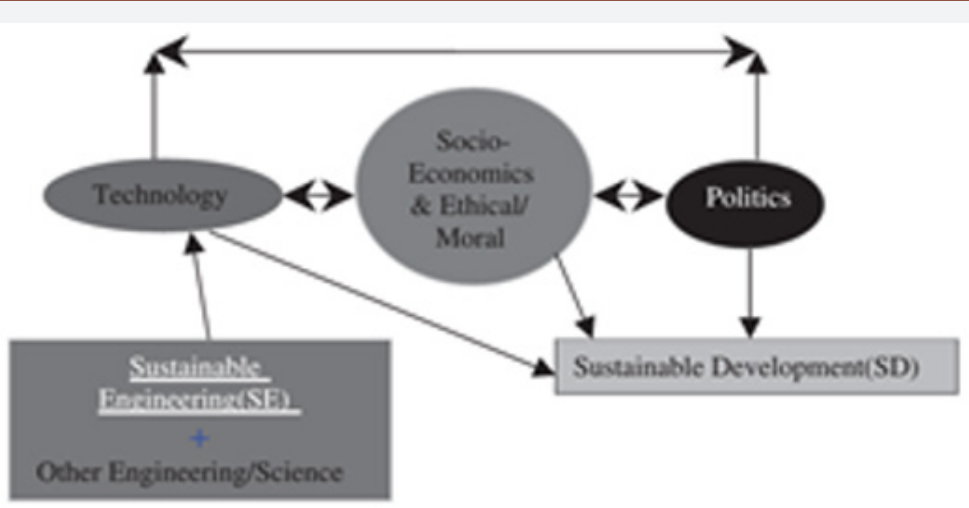

Figure 2: Subsystem of sustainable Development (SD).

Note: SE is a subsystem of technology (or SDE), which is a subsystem of SD. An example of technology politics interaction is the U.S. Department of Energy's extensive research for the efficient production of hydrogen and cellulosic bioethanol after the state of the union addresses by President Bush in 2003 and 2006. Engineers should focus on SDE within the framework of other components.

- Extending producer responsibility for the lifecycle environmental impacts of goods and services

- Introducing extensive eco-labeling programs

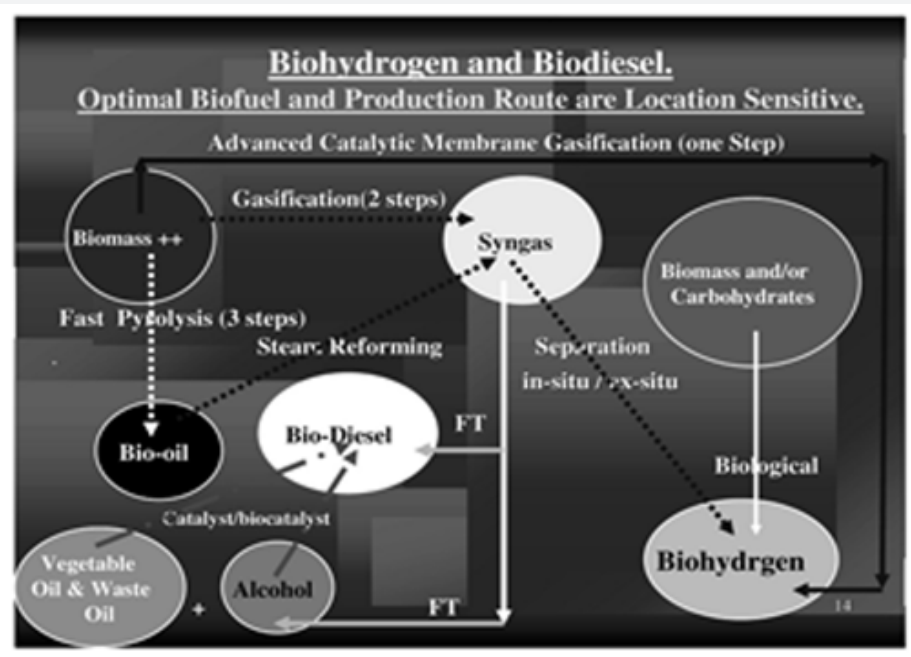

Figure 3: Some Routes to Bio- Diesel and Bio- Hydrogen. Note: FT-Fisher Tropsch

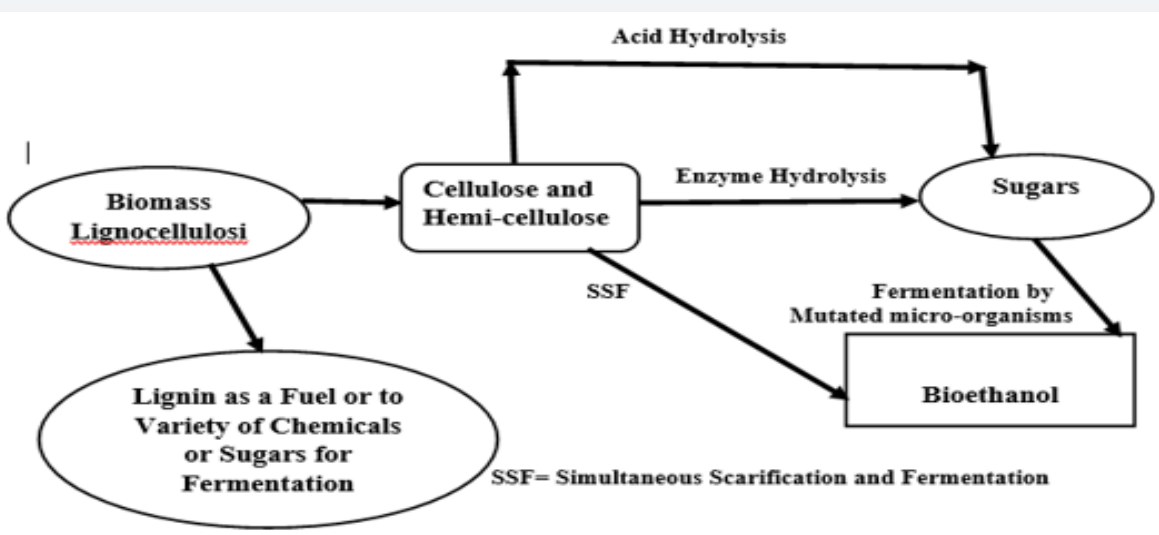

Figure 4: Biochemical Routes to Bioethanol. Critical Evaluation of the different Biochemical Routes to Bioethanol from Biomass. 
- $\quad$ Clear definitions for Energy Ratio (ER) and Net Energy Ratio (NER) (see http://www.energy.gov/, http://www.ers. usda.gov/) should be developed to clear the present confusion regarding ER $<1.0$ and NER $<0.0$ and Quality of Energy (QE).

To simplify the calculations as shown below, let us define the terms as follows:

Energy of fuel=a, Energy consumed to produce the fuel=b=X and $\mathrm{Y}=\mathrm{a}-\mathrm{b}$

A brief review of them can be given in the following few lines: ER of a fuel is defined as (Energy of Fuel)/ (Energy Consumed to Produce the Fuel) $=a / b$, while NER of a fuel is energy consumed per energy produced $=[($ Energy of Fuel - Energy Consumed to Produce the Fuel) (+/-) Energy Consumed to Produce the Fuel)]/ (Energy Consumed to Produce the Fuel $)]=[\mathrm{Y}(+/-) \mathrm{X}] / \mathrm{X}$. The $(+/-)$ sign is defined as follows: It is +when $Y$ is positive $(E R>1.0)$ and - when $\mathrm{Y}$ is negative $(\mathrm{ER}<1.0) . \mathrm{Y}=$ [Energy of Fuel - Energy Consumed to Produce the Fuel $]=a-b$ as shown above.

Examples

a. One researcher gave the following: Energy used to produce ethanol $(b=X)=78,081.00 \mathrm{Btu} / \mathrm{gal}$, energy of ethanol (a) $=83,961.00 \mathrm{Btu} /$ gal, thus, $\mathrm{Y}=\mathrm{a}-\mathrm{b}=+5,880.0$ and the above sign is + and NER $=(5,880.0+78,081) / 78,081=1.0753$ and $\mathrm{ER}=1.0753$. Meaning: If $100.0 \mathrm{~kJ}$ is consumed to produce an amount of ethanol, this ethanol will contain $107.53 \mathrm{~kJ}$ of energy.

b. Another researcher gave the following: Energy used to produce ethanol using different technology and feedstock(b) $=131,017 \mathrm{Btu} /$ gal, energy of ethanol $(\mathrm{a}=\mathrm{X})=76,000 \mathrm{Btu} /$ gal, thus, $\mathrm{Y}=-55,017.00$ and the above sign is - and NER $=(-55,017.00$ - 131,017)/131,017 =-1.42 and ER =76,000/131,017 =0.58. Meaning: If $100.0 \mathrm{~kJ}$ is consumed to produce an amount of ethanol, this ethanol will contain $58 \mathrm{~kJ}$ of energy (but may be of higher QE). Higher QE for the same amount of energy is described by some researchers as related to the ease of energy transformation, energy which is easier to transform is of higher QE. The above examples show how sensitive the calculation of ER and NER is and also how the QE can affect the situation. The main differences in calculating ER and NER are as follows:

- $\quad$ Energies included: Some researchers include solar energy used in the production of the raw materials for the production of the fuel, which is quite disputable.

- Levels of technologies: Some researchers use older technologies when computing energy consumed to produce the fuel, which is not correct.

- Energy estimation techniques: Different researchers use different estimation techniques, which create artificial differences. These estimation techniques need to be standardized.

- Energy is included/not included in capital cost and estimation techniques.
- Energy credits for by-products are included/not included.

Data about ER and NER from the US Department of Agriculture (USDA) (http://www.ers.usda.gov/) and US Department of Energy (DOE) (http://www .energy.gov/) are given in Table 1. It is clear that bio - ethanol is the only fuel on the list with ER $>1.0$ and NER $>0.0$. Other fuels with ER $<1.0$ and NER $<0.0$ are valid fuels simply because energy of fuel is of higher QE than energy consumed to produce the fuel, for example, electricity from coal (ER =0.4, NER =-1.6). The cellulosic bio - ethanol will be even better than the present corn bio - ethanol as shown in (Table 1\&2).

Table 1: Energy Ratio (ER) and Net Energy Ratio (NER) from the U.S. Department of Agriculture, U.S. Department of Energy and Other Sources. Examples from the U.S. Department of Agriculture (supporting ethanol from corn).

\begin{tabular}{|c|c|c|}
\hline Fuel & ER & NER \\
\hline Gasoline & 0.8 & -1.2 \\
\hline Diesel & 0.8 & -1.2 \\
\hline Electricity & 0.4 & -1.6 \\
\hline Bio - ethanol & 1.6 & 1.6 \\
\hline Natural gas & 0.9 & -1.1 \\
\hline $\begin{array}{c}\text { Liquefied Petroleum } \\
\text { Gas(LPG) }\end{array}$ & 0.95 & -1.05 \\
\hline Coal & 0.95 & -1.05 \\
\hline
\end{tabular}

Table 2: Corn and Cellulosic Bio - Ethanol.

\begin{tabular}{|c|c|c|}
\hline & ER & NER \\
\hline Ethanol from corn & $1.25-1.35$ & $1.25-1.35$ \\
\hline Ethanol from cellulosic waste & 1.8 & 1.8 \\
\hline
\end{tabular}

- $\quad$ Final energy utilization is a subsystem of SDE and SD, e.g.: utilization of fuel/ bio-fuel cells and/or integrated electrolysis (bio-electrolysis), and efficient hydrogen/oxygen storage using nanotechnology to efficiently transform intermittent solar energy into a continuous electric supply in auto-thermal housing see (http://www3.imperial.ac.uk/fuelcells/reports1/ conference\%20reports). Electrolysis and fuel cells are basically the opposite of each other, thus producing hydrogen by electrolysis in order to utilize it for the production of electricity in fuel cells is certainly not a good idea from an energy point of view. However, it can be used to turn intermittent solar energy to continuous electric energy for many applications including auto-thermal housing. Bio-electrolysis is in fact electrolysis assisted by the biochemical power of micro-organisms to break $\mathrm{H}_{2} \mathrm{O}$ molecules in the electrolyte solution. It can also be considered as bio-hydrogen production by a micro-organism assisted by the ability of electric current to break down the $\mathrm{H}_{2} \mathrm{O}$ molecules. The bio-fuel cell can have two sides of its bio-action: at the anode, the microorganisms assisting in the ionization of the hydrogen molecules, and at the cathode, using ferric ions instead of oxygen, and when it is reduced to ferrous ions, it is regenerated to ferric ions using a bioreactor, which does that while utilizing $\mathrm{CO}_{2}$ for photosynthesis to grow the microorganisms and produce a single-cell protein for animal 
feed . Optimal design should also be applied for integral system where the bio-electrolyzer can be operated on electrical power from solar energy to produce hydrogen and the entire system should be net energy producer by supplying the bio-fuel cell by hydrogen during the sunny morning and excess hydrogen to be stored and used to supply the bio-fuel cell by night. Novel techniques for hydrogen storage using nanotechnology are developed and are very successful. For example, carbon nanotubes; and a flexible bio-electrolyzer, which operates in the morning for electrolysis and by night as an additional bio-fuel cell, will represent a considerable addition to the productivity of this integral bio-system.

- $\quad$ BFs are sensitive to many parameters; the most important are: location; type and source of RRMs and size of investment/ market. These simple facts imply the following:

a. no single BF will dominate the market, in other words: the present matrix of dirty, non-renewable fuels will be replaced by a matrix of clean, renewable fuels

b. no single technology for the same BF will dominate; each RRM and each region will suggest its optimum technology and it will also develop with time and MD research

- The present situation of BFs production from agriculture products (e.g., bio-ethanol from corn and bio-diesel from vegetable oils, etc.), especially in the Mid-East of the USA (relying on corn itself as a feedstock) is not a strategic, longterm, large-scale solution for the renewable energy problem for SDE. It will be replaced by large-scale production of bio - fuels from waste (e.g., cellulosic bio - ethanol, FT biodiesel, etc.). But unfortunately, this BFs production from food resources will still occupy a small part of the matrix of feedstock for BFs.

\section{SD, SDE, and EE}

Many SD definitions are not scientific, especially those emerging from politically oriented United Nation (UN) political conferences. An example is: "It is the form of technological, economic and social strategy for development that provides a mode of development so that future generations will have at least the same opportunities to live and prosper that the present generation enjoys". Does this mean that we are happy with the present situation, locally, statewise, or world-wide? The answer by any intelligent-consciousness person should be no, the \% happy with the present situation is very small, and their activities are harmful to the majority of the people, to the earth, its environment and are opposite to SD its definition should be more concrete and specific than that. Definition should be formed of a number of components and not catch false phrases like the above.

The healthy and constructive definition should involve:

- Being against war, destruction, weapons of mass destruction and violation of human rights.

- $\quad$ Being against all kinds of exploitation of countries against each other and humans inside the same country against each other
- Concentrate on developing novel clean technologies capable of achieving minimum pollution-maximum production.

- $\quad$ Use agricultural waste and other RRMs to produce BFs and BPs and concentrate on the use of the most advanced IBRs.

- Do not concentrate only on profit but develop technologies capable of using RRMs to achieve SD.

- Adoption of international policy of the use of natural distributed RRMs in contradistinction to the present depletion of non-RRMs concentrated in certain parts of the world causing conflicts, wars and exploitation of the non-RRMs producing countries by industrialized military powerful countries.

- $\quad$ Focusing on the production of environmentally friendly products easy to degrade and/or reuse and fight bad consumption habits which are environmentally un-friendly.

- $\quad$ Make best use of waste, e.g.: Municipal Solid Waste (MSW) to produce energy utilizing latest most efficient technology of Bio-Reactors-Land - Fills (BRLFs). The same for other wastes and recycling.

- Develop clean/low emission, environmentally friendly housing, office buildings, and shopping centers.

- Developing political and socio-economic structures to be applied to the above sides and include nonprofit organizations to help these processes and help to change consumer habits.

- Developing IBRs for the production of BFs/BPs using novel technologies and RRMs.

As explained earlier SD is the larger system and SDE is a subsystem of it and EE is a subsystem of SDE. RRMs is the other subsystem that with EE forms SDE. The most important class of RRMs addressed in this paper is the lingo-cellulosic waste from different sources the main one being the agricultural waste.

\section{BFs, BPs and IBRs}

IBR is a complex facility that integrates different biomass conversion processes and equipment to produce BFs, BPs and power. In a sense it is analogous to today's petroleum refineries and its integrated petrochemical complexes, which produce multiple fuels and products from petroleum, coupled to other chemical plants. IBRs are the most promising route to the creation of a new domestic and distributed bio-based industry. SD does not depend only on sustainable BFs but also on sustainable BPs. This important simple fact leads to the important concept of IBRs producing not only sustainable BFs but also other sustainable BPs and energy. The National Renewable Energy Laboratory (NREL), which is a part of the US DOE, defines bio-refinery as: "a facility that integrates biomass conversion processes and equipment to produce fuels, power, and chemicals from biomass." The present view about IBRs is based on two (or more) platforms, the sugar platform and syngas platform and possibly other platforms such as the algae to diesel platform as discussed later. These two (or more) platforms distinguish it from EBRs, which are subsystems of IBRs. Facilities for 
production of BFs are subsystems of IBRs or EBRs. The implications of this are as follows:

An EBR built today to produce BFs should be planned to grow into IBR, for BFs production will always be a subsystem of IBRs. Clear definitions of IBRs and their subsystems of EBRs and the related BFs and BPs and power should be well developed and related to each other. Both the National Science Foundation (NSF) and DOE in the USA and similar organization in Europe and the rest of the world are putting large research funds into development of IBRs and its subsystems. Bio-Reactors (BRs) are integral critical subsystems of $\mathrm{SD}$, which is MD by its very nature, as discussed earlier. It is best to use the ISA to study this complex MD system and its subsystems. Engineers are most interested in the technology part (subsystem of the SD system) but with a background of other subsystems and MD collaboration as discussed earlier. The non-linear relations between SD, SDE, EE and RRMs were presented simply and briefly above. RRMs should be renewable over a period of 6 to 18 months. RRMs can be any kind of renewable waste, for example, agricultural waste, municipal waste, and so on, or special (energy) crops produced specifically to be used for this purpose, for example, switch-grass cultivated and produced especially for BFs production. On the other hand, useful agricultural products used today for BFs, for example, corn for ethanol, vegetable oil for trans-esterification to biodiesel, and so on, are not strategic solutions as RRMs for SD because of the consumption of important edible products at a time of food shortage everywhere, especially in developing nations. Renewable biomass, the main group of RRMs, is a storage tank for solar energy through biosynthesis, $\mathrm{CO}_{2}$ and other nutrients. The ultimate aim for BFs is to produce as much $\mathrm{CO}_{2}$ as consumed in the biosynthesis of the biomass that produced it. This, with maximum efficiency, may lead to approaching zero net $\mathrm{CO}_{2}$ emission. Despite all claims and end of pipe treatments we cannot really dispose of all $\mathrm{CO}_{2}$ resulting from fossil fuel; it only keeps circulating from one form/ place to the other, except with sequestration by injection under the bottom of the ocean, which is expensive, applicable to large scale rich companies and may be dangerous. All other techniques just move $\mathrm{C}$ from one location/form to the other without reducing the earth $\mathrm{C}$ overall inventory from the carbon source that came from under the ground. Hydrogen is a good clean fuel and will occupy its part in the clean fuels matrix and is, therefore, one of the potential products of IBRs. However, although the claim that it is $100 \%$ clean is locally true, it is actually not globally true if its source is fossil fuel, whether directly through Catalytic Steam Reforming (CSR) (95\% of hydrogen produced in USA is through CSR) or indirectly through use of electricity ( $90 \%$ of electricity in the USA is from coal) by electrolysis. In both cases the production of CO and C during CSR and production of electricity make it not globally clean. It can only be globally clean if the source is not fossil fuel, e.g.: bio, wind, solar, hydro, nuclear, etc. Back to the biochemical route, it is important to point out, as will be shown below, that the range of sugars that can be fermented to useful BFs like bio-ethanol is expanding due to the development in microbiology and the discovery of efficient mutated microorganisms capable of fermenting the wide range of sugars produced from cellulose/hemicellulose hydrolysis. Also the novel designs and modes of operations of fermenters open the doors wide open for increase in the efficiency and productivity of bio-ethanol. Additional strong challenge with lots of intensive research nowadays is in the field of efficient and clean hydrolysis of cellulose/hemi-cellulose; important advancements are achieved in the field of enzymatic hydrolysis of cellulose/hemi-cellulose. Important improvements are introduced to syngas production from biomass through both a one-step process (biomass gasification to syngas) and a two-step process (biomass fast pyrolysis to bio-oil followed by CSR of bio-oil to syngas). The two-step process seems to be better with regard to the percentage of $\mathrm{H}_{2}$ in syngas. Certain difficulties associated with CSR of bio-oil are solvable through novel reformers configurations such as Circulating Fluidized Bed (CFB). An important challenge in the biomass to FT bio-fuels process (Figure 3) is the integration of the endothermic CSR process with the exothermic catalytic FT process into one integrated membrane catalytic auto-thermal process. IBRs should contribute towards SD and not only produce BFs; they should also include all that is bio, whether with regard to feedstock, processes, or both, for instance:

- $\quad$ Biomass utilizing sequential thermal catalytic processes to produce FT bio - diesel (the processes are not bio, but the feedstock is bio)

- $\quad$ Biological treatment of CO and water to produce C and hydrogen (bio-catalyzed Water Gas Shift [WGS] reaction; the feed is not bio, but the process is bio).

- $\quad$ Utilizing lignocelluloses to produce cellulosic bio - ethanol (both feedstock and processes are bio).

- Utilization of bio - processes to transfer one form of energy to another [e.g.: Integrated Bio-Electrolysis coupled to Bio-Full-Cells (IBE-BFCs)] used to change intermittent solar energy into continuous electric energy, which is useful for many applications including auto-thermal housing.

IBRs can either grow with time as parts of existing plants (e.g., pulp and paper industry) or start as an IBR based on BFs production and grow into a complete IBR. Other forms of RCE (e.g., wind energy) can be integrated with the BFs to generate the total renewable energy profile of an IBR. Central intermediates (platforms) for the BFs part of IBRs are sugars suitable for fermentation to bioethanol and/or bio-butanol and syngas suitable for both hydrogen extraction and use in the FT process for producing fuels from as low as methanol up to diesel. It is possible to combine gasification of biomass (or fast pyrolysis followed by CSR of the product biooil) to produce bio-syngas with the fermentation of this bio-syngas to produce bio-ethanol and or bio-butanol. Direct utilization of solar energy through different direct techniques (e.g., photocells) without passing through the biosynthesis stage is a possibility, but it has its own bottlenecks and should be integrated with other technologies (e.g., IBE-BFCs) to fully utilize it as a part of autothermal housing and IBRs. From the above, it is clear that neither single BF nor single technology will dominate. Humanity will move 
from today's matrix of dirty, non-renewable fuels to a future matrix of clean, renewable fuels, with the sub-matrix of different BFs from different RRMs-technologies occupying a large portion of the clean fuels matrix, and that SD will not be achieved through BFs only but should be extended to IBRs, combining BFs and BPs with efficient bio-energy in order to achieve SD. The status of today's IBR concept can be summarized as shown in Figure 5, and the future view of a self-sustained clean town, as shown in Figure 6 (Figure 5).

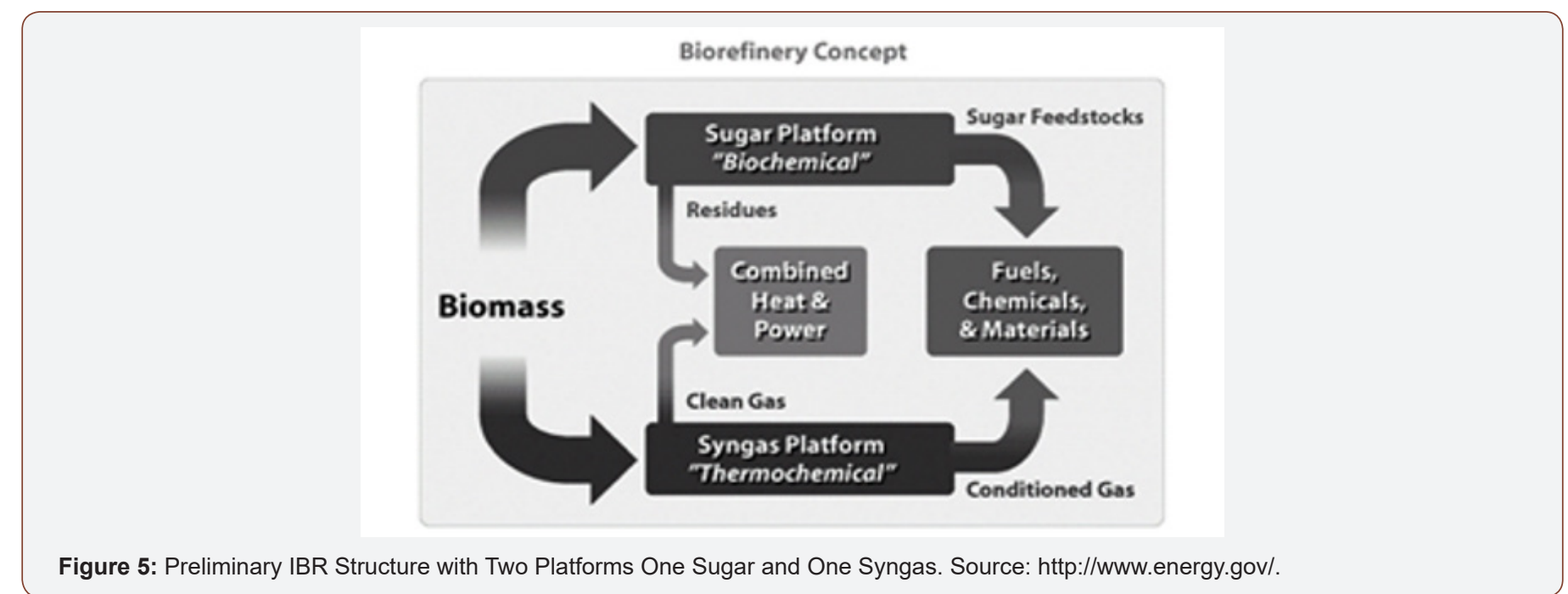

Open political, socio-economical, and moral/ethical questions

Some questions are offered here for thinking and discussion, such as:

- Is SD profitable or is it a moral/ethical obligation coupled to continuation of future development?

- Is there a contradiction between SD and profitability? And if there is, is it solvable within a profitability-based society? And how?

- $\quad$ Are BFs, BPs/IBRs enough to achieve SD? If not, what else is needed?
- Will SD affect international relations, decreasing international tensions and wars?

- Is SD compatible or contradictory to globalization?

- What level of public awareness is needed to adopt SD policies and what are the best techniques to achieve that? Is this a political or a socio-economical question?

- Do world politics and large incorporated businesses affect adaptation of BFs, BPs and IBRs?

- What is the relation between wars and SD? Will adaptation of SD decreases wars?

- Is nuclear energy a good and promising option for SD?

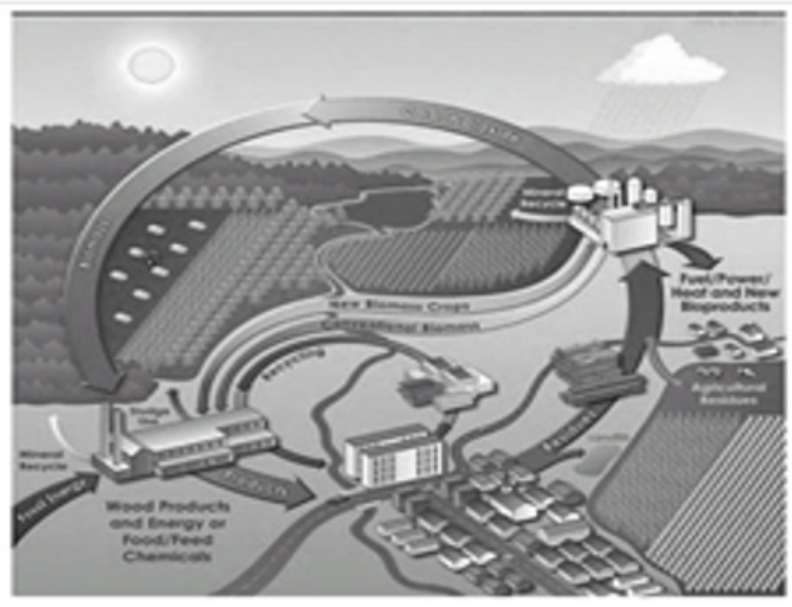

Figure 6: Sustainable and Clean Future Town. Source: http://www.energy.gov/.

- Is there a contradiction between SD and the second law of thermodynamics? (Figure 6)

\section{Partial Conclusion}

From the above descriptive discussion, it is essential to realize that SD is crucial for the development of human societies and that 
it is MD by its very nature. The presented tool of ISA based on ST is the best to study and develop the different subsystems of this complex MD system. For engineers, the most important subsystem is the technology one and SDE is a subsystem of it. BFs is one of the most important subsystems of SDE, however, their development is not sufficient for SD; other BPs bio - energy from RRMs are essential, leading to the concept of IBRs, which is very important for SD. BFs and BPs represent a subsystem of IBRs. Well-directed innovative research in all subsystems of IBRs is essential, taking into consideration the other subsystems of SDE as well as the nontechnical subsystems of SD.

\section{Acknowledgement}

None.

\section{Conflict of Interest}

No conflict of interest. 